\title{
An Asthma Protocol Improved Adherence to Evidence-Based Guidelines for Pediatric Subjects With Status Asthmaticus in the Emergency Department
}

\author{
Andrew G Miller RRT-ACCS, Moira E Breslin MD, Leslie C Pineda MD, and James W Fox MD
}

\begin{abstract}
BACKGROUND: In our institution's pediatric emergency department, adherence to evidencebased asthma guidelines was noted to be suboptimal for patients with asthma exacerbations. We hypothesized that an evidence-based asthma protocol would improve time to treatment and adherence to National Institutes of Health guidelines for patients presenting to the emergency department with status asthmaticus. METHODS: Subjects at our institution were retrospectively identified through an electronic medical record search following institutional review board approval. The asthma protocol was initiated in February 2012. All pediatric subjects who received continuous albuterol in the emergency department before (February 26, 2009, to February 22, 2012, $n=193$ ) and after (February 23, 2012, to December 31, 2012, $n=68$ ) protocol initiation were analyzed. The post-protocol data were collected as part of routine quality assurance monitoring with a target of 60 post-protocol subjects. Subjects were identified at the end of each month, which resulted in a total of 68 subjects being included. Primary outcomes measured included time to initial treatment with inhaled bronchodilator therapy, time to treatment with systemic corticosteroids, and total number of ipratropium bromide treatments delivered. RESULTS: Two-hundred sixty-one subjects $(7.1 \pm 4.6 \mathrm{y}$ of age, $66 \%$ male) were included. Demographics were similar in the pre- and postprotocol groups. Compared with the pre-protocol group, more subjects in the post-protocol group received bronchodilators within $30 \mathrm{~min}(60 \%$ vs $77 \%, P=.02)$, at least one dose of ipratropium bromide (55\% vs $87 \%, P<.001), 3$ doses of ipratropium bromide $(14 \%$ vs $54 \%, P<.001)$, and corticosteroids within 60 min $(62 \%$ vs $77 \%, P=.04)$. There were no statistically significant differences between the pre- and post-protocol cohorts in the mean time to first bronchodilator treatment ( $32 \pm 41$ vs $26 \pm 52 \mathrm{~min}, P=.34)$, mean time to corticosteroid administration $(74 \pm 68$ vs $54 \pm 63 \mathrm{~min}, P=.06)$, or mean emergency department length of stay ( $342 \pm 143$ vs $364 \pm 183 \mathrm{~min}$, $P=.31)$. CONCLUSIONS: An asthma protocol resulted in improved adherence to National Institutes of Health guidelines in children with status asthmaticus and improved efficiency in the administration of rescue bronchodilator and systemic corticosteroid therapy. Key words: pediatric asthma; asthma; status asthmaticus; asthma protocol; protocol-based care; severe asthma; continuous bronchodilator; emergency department. [Respir Care 2015;60(12):1759-1764. (c) 2015 Daedalus Enterprises]
\end{abstract}

Introduction

Asthma exacerbations are a common reason for pediatric patients to seek care in an emergency department, with an estimated $20 \%$ of children with asthma in the United

\footnotetext{
Mr Miller is affiliated with the Department of Respiratory Care Services, and Drs Pineda and Fox are affiliated with the Department of Pediatrics, Duke University Medical Center, Durham, North Carolina. Dr Breslin is affiliated with the Department of Pediatrics, University of California at Los Angeles, Los Angeles, California.
}

States requiring emergency department visits annually due to exacerbations. ${ }^{1-3}$ Bronchodilator and systemic cortico-

\footnotetext{
This study was supported by departmental funding. The authors have disclosed no conflicts of interest.

Correspondence: Andrew G Miller RRT-ACCS, Respiratory Care Services, Duke University Medical Center, PO Box 3911, 2301 Erwin Road, Durham. NC 27710. E-mail: andrew.g.miller@duke.edu.
}

DOI: $10.4187 /$ respcare.04011 
steroid therapies are the mainstays of emergency department management of asthma exacerbations. Despite appropriate emergency department treatment, up to $27 \%$ of these patients require admission, making asthma the most common reason for pediatric hospitalization. ${ }^{3}$ Status asthmaticus represents an important subset of pediatric asthma exacerbations, with nearly 500,000 patients requiring admission to the pediatric ICU each year. ${ }^{3}$

An analysis of our institution's emergency department asthma management revealed that adherence to evidencebased asthma guidelines was suboptimal. Areas identified for potential improvement are detailed in Figure 1. In particular, 3 weaknesses were identified: (1) extended time to corticosteroid administration, (2) inadequate ipratropium administration, and (3) inconsistent adherence to National Institutes of Health (NIH) recommendations ${ }^{4}$ for bronchodilator administration (specifically, 3 treatments with albuterol and ipratropium bromide every $20 \mathrm{~min}$ ). A multidisciplinary team of physicians, nurses, respiratory therapists (RTs), and emergency department pharmacists designed a nurse-initiated pediatric asthma treatment protocol to address these deficiencies. This protocol was based on the 2007 NIH guidelines and designed to be nurseinitiated, as triage nurse-driven protocols have been shown to reduce hospital admissions, time to corticosteroid administration, time to patient improvement, and time spent by patients in the emergency department. ${ }^{4-6}$ The effect of a nurse-driven protocol in the emergency department setting on patients with status asthmaticus has not been studied. The objective of this study was to examine the effects on time to initial treatment and adherence to evidencebased practice for status asthmaticus after the implementation of an evidence-based, nurse-initiated pediatric asthma protocol in our pediatric emergency department.

\section{Methods}

\section{Subjects}

Subjects 2-17 y olds with a known history of asthma, wheezing, or reactive airway disease who presented with signs and symptoms of an asthma exacerbation were first evaluated by a triage nurse for protocol eligibility. The triage nurse measured vital signs, including pulse oximetry; performed a brief history and physical examination in assessment rooms specifically dedicated to this process; calculated the modified pulmonary index score; and initiated the standing order set if the modified pulmonary index score was $\geq 6$ (moderate-to-severe exacerbations). As part of the triage process, subjects were assigned a triage priority based on how urgently they needed to be seen, with 1 being the most urgent. The modified pulmonary index score was chosen as a practical alternative to serial measurement of disease severity via bedside spirometry or

\section{QUICK LOOK}

\section{Current knowledge}

Asthma exacerbations are a common reason pediatric patients seek care in an emergency department, with an estimated $20 \%$ of children with asthma in the United States requiring emergency department visits annually. Bronchodilator and systemic corticosteroids are the mainstays of emergency department management. Despite appropriate treatment, up to $27 \%$ of these patients require admission, making asthma the most common reason for pediatric hospitalization.

\section{What this paper contributes to our knowledge}

An asthma protocol instituted in an emergency department resulted in improved adherence to National Institutes of Health guidelines in children with status asthmaticus and improved efficiency in the administration of rescue bronchodilator and systemic corticosteroid therapy. More subjects in the protocol group received bronchodilators within $30 \mathrm{~min}$, at least one dose of ipratropium bromide, the recommended 3 doses of ipratropium bromide, and received corticosteroids within $60 \mathrm{~min}$.

peak-flow monitoring because many pediatric patients are unable to perform reliable measurements during an exacerbation. ${ }^{2}$ The modified pulmonary index score is known to have high inter-observer correlation among nurses, physicians, and $\mathrm{RTs}^{7}$ while providing a shared resource to communicate severity of asthma exacerbations. The order set directs nurses to initiate continuous pulse oximetry, administer oral prednisolone, and contact the RT to administer inhaled albuterol and ipratropium bromide.

We studied subjects with severe asthma exacerbations (defined as those ill enough to require continuous albuterol therapy) because these children are the most likely to develop respiratory failure, require ICU admission, or acutely decompensate if they are not identified early and treatment is delayed. In our emergency department, continuous albuterol therapy is reserved for patients with severe respiratory distress or those who have failed to respond sufficiently to initial intermittent bronchodilator therapy. There are no specific criteria for continuous albuterol therapy. Prior analysis of data from our institution found that those treated with continuous albuterol were among the most ill, with $90 \%$ ultimately requiring hospital admission for further management. ${ }^{8}$ Asthma subjects requiring continuous albuterol therapy were retrospectively identified through an electronic record search of respiratory care service documentation following an institutional review boardapproved protocol. 


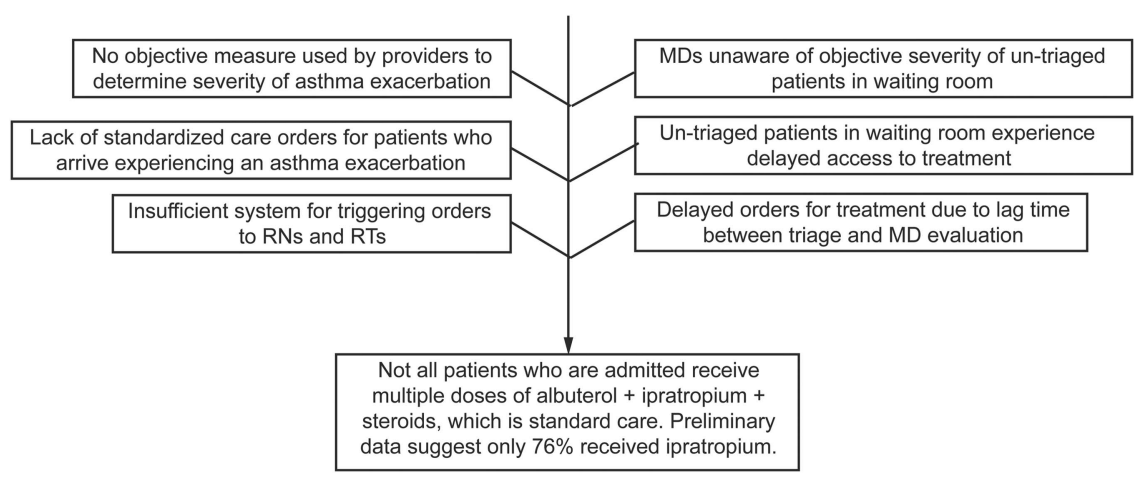

Fig. 1. Analysis of the need for asthma protocol. $\mathrm{MD}=$ medical doctor; $\mathrm{RN}=$ registered nurse; $\mathrm{RT}=$ respiratory therapist.

The pediatric asthma protocol was initiated on February 23, 2012. All children who received continuous albuterol in the emergency department before (from February 26, 2009, to February 22, 2012) and after (from February 23, 2012, to December 31, 2012) protocol initiation were analyzed. The pre-protocol subjects were identified from an existing research data set. The post-protocol data were collected as part of routine quality assurance monitoring, with a target of 60 post-protocol subjects. Subjects were identified at the end of each month, which resulted in a total of 68 subjects being included.

Primary outcomes included time to initial treatment with inhaled bronchodilator therapy and systemic corticosteroids, adherence to NIH guidelines for ipratropium bromide administration, and total time spent in the emergency department. We defined the following treatment time end points a priori. (1) Time to first bronchodilator administration was $30 \mathrm{~min}$ from the initial nursing evaluation. This allowed adequate time for identification of an asthma exacerbation, placement of the subject in a treatment room, initiation of the standing order set, notification of the RT, and the logistics of medication administration. (2) Time to administration of systemic steroids was $60 \mathrm{~min}$ from the first nursing evaluation. This time frame was chosen as receiving corticosteroids within $60 \mathrm{~min}$ of arrival has been found to reduce admission rates for asthma exacerbations. ${ }^{9}$

\section{Statistical Analysis}

Data were analyzed with statistics software from GraphPad Software (San Diego, California). Continuous data are presented as mean \pm SD. Pre- and post-protocol values were compared with the unpaired $t$ test for continuous variables and the Fisher exact test for categorical data as appropriate. $P<.05$ was considered statistically significant.

\section{Results}

Two-hundred sixty-one subjects were included (7.1 \pm 4.6 y of age, $66 \%$ male): 193 in the pre-protocol
Table 1. Demographic and Clinical Variables for the Pre- and PostProtocol Groups

\begin{tabular}{lccc}
\hline \hline \multicolumn{1}{c}{ Variable } & $\begin{array}{c}\text { Pre-Protocol } \\
(n=193)\end{array}$ & $\begin{array}{c}\text { Post-protocol } \\
(n=68)\end{array}$ & $P$ \\
\hline Age, mean \pm SD y & $7.1 \pm 4.8$ & $7.1 \pm 3.9$ & $>.99$ \\
Males, $n(\%)$ & $125(65)$ & $48(71)$ & .46 \\
Arrived via emergency medical & $86(45)$ & $19(28)$ & .02 \\
$\quad$ services, $n(\%)$ & & & \\
Triage priority, mean \pm SD & $2.4 \pm 0.6$ & $2.4 \pm 0.6$ & $>.99$ \\
Highest triage priority (1-2), $n(\%)$ & $116(60)$ & $44(65)$ & .56 \\
Heliox, $n(\%)$ & $26(14)$ & $9(13)$ & $>.99$ \\
Intravenous magnesium, $n(\%)$ & $82(43)$ & $36(53)$ & .16 \\
Admitted, $n(\%)$ & $175(91)$ & $64(95)$ & .46 \\
Pediatric ICU, $n(\%)$ & $66(34)$ & $29(43)$ & .24 \\
Initial modified pulmonary & NA & $10.9 \pm 2.4$ & NA \\
$\quad$ index score & & & \\
& & & \\
NA = not available & & & \\
& & & \\
\hline
\end{tabular}

cohort and 68 in the post-protocol cohort. There were no statistically significant differences between groups for age, sex, or triage priority. The modified pulmonary index score was implemented as part of the protocol and was not recorded pre-protocol. Compared with the pre-protocol cohort, the post-protocol cohort was less likely to arrive via emergency medical services ( $45 \%$ vs $28 \%, P=.02$ ). Overall, $58(85 \%)$ of the post-protocol subjects were eligible for the protocol, and of these, $60 \%$ had the protocol activated. Only $22 \%$ had the protocol initiated in triage, as had been designed in the protocol. There was no difference between groups for heliox (helium-oxygen mixture) use (14\% vs $13 \%, P>.99$ ), intravenous magnesium administration $(43 \%$ vs $53 \%, P=.16)$, overall admission rate ( $91 \%$ vs $95 \%, P=.46)$, or need for pediatric ICU admission $(34 \%$ vs $43 \%, P=.24)$ (Table 1$)$.

\section{Bronchodilator Administration}

There was no difference between the pre- and postprotocol cohorts in the mean time to initial bronchodilator 
Table 2. Outcomes Following Asthma Protocol Initiation for Administration of Medications Following NIH Guidelines

\begin{tabular}{|c|c|c|c|}
\hline Variable & Pre-Protocol $(n=193)$ & Post-Protocol $(n=68)$ & $P$ \\
\hline \multicolumn{4}{|l|}{ Results for bronchodilator administration } \\
\hline Time to initial bronchodilator administration, mean $\pm \mathrm{SD}$ min* & $32 \pm 41$ & $26 \pm 52$ & .34 \\
\hline Inhaled bronchodilator received within $30 \mathrm{~min}, n(\%)$ & $116(60)$ & $52(77)$ & .02 \\
\hline Received at least 1 dose of ipratropium bromide, $n(\%)$ & $107(55)$ & $59(87)$ & $<.001$ \\
\hline Received 3 doses of ipratropium bromide, $n(\%)$ & $26(14)$ & $37(54)$ & $<.001$ \\
\hline Placed directly on continuous albuterol, $n(\%)$ & $69(36)$ & $7(10)$ & $<.001$ \\
\hline \multicolumn{4}{|l|}{ Results for corticosteroid administration } \\
\hline Received prior to arrival, $n(\%)$ & $37(19)$ & $14(21)$ & .86 \\
\hline Time to corticosteroid administration, mean \pm SD min* & $74 \pm 68$ & $54 \pm 63$ & .06 \\
\hline Received within 60 min while in emergency department, $n(\%)$ & $83 / 156(53)$ & $38 / 54(70)$ & .04 \\
\hline
\end{tabular}

* All times except time spent in the emergency department were determined from triage time, which was determined from registration time. Time to corticosteroid administration included only subjects who received corticosteroids in our emergency department.

treatment post-protocol implementation $(32 \pm 41 \mathrm{vs}$ $26 \pm 52 \mathrm{~min}, P=.34)$. There were, however, significant improvements between the pre- and post-protocol cohorts in the number of subjects who received bronchodilators within the a priori cutoff of $30 \mathrm{~min}(60 \%$ vs $77 \%, P=.02)$, at least one inhaled dose of ipratropium bromide (55\% vs $87 \%, P<.001)$, the NIH-recommended 3 doses of ipratropium bromide ( $14 \%$ vs $54 \%, P<.001)$, and immediate (without receiving any intermittent treatments) administration of continuous albuterol (36\% vs $10 \%, P<.001)$.

\section{Systemic Corticosteroid Administration}

Subjects who received systemic corticosteroids before emergency department arrival were excluded from timeto-treatment analysis (37 [19\%] pre-protocol subjects and 14 [21\%] post-protocol subjects). The improvement in the mean time to corticosteroid administration was not statistically significant $(74 \pm 68$ vs $54 \pm 63 \mathrm{~min}, P=.06)$. However, there was a statistically significant improvement in the number of subjects receiving corticosteroids within the a priori cutoff of $60 \mathrm{~min}$ from triage evaluation $(53 \%$ vs $70 \%, P=.04$ ) (Table 2 ). There was no significant difference between the pre- and post-protocol cohorts for the mean time spent in the emergency department $(342 \pm 143$ vs $364 \pm 183 \mathrm{~min}, P=.31)$.

\section{Discussion}

To our knowledge, this is the first study to specifically assess the impact of an evidence-based, nurse-initiated asthma protocol for children with status asthmaticus in a pediatric emergency department. Importantly, our study demonstrated that in subjects with very severe exacerbations (ie, requiring continuous albuterol therapy), this protocol improved the efficiency of rescue bronchodilator and systemic corticosteroid therapy delivery.
Our results are consistent with those of Qazi et al, ${ }^{10}$ who found that time to first salbutamol administration (mean of $31 \mathrm{~min}$ ) and time to corticosteroid administration improved after their emergency department implemented a nurseinitiated protocol. Similar to our study, Qazi et al failed to find a reduction in emergency department length of stay despite earlier implementation of appropriate rescue medications. However, unlike the work of Oazi et al, our study was designed to evaluate medication administration efficiency and adherence to evidence-based guidelines.

Zemek et $\mathrm{al}^{6}$ demonstrated improvement in emergency department length of stay and admission rate after implementation of a nurse-initiated protocol for corticosteroid administration for pediatric asthma exacerbations. Importantly, however, their institution had $10 \mathrm{y}$ of experience using a nurse-driven bronchodilator administration protocol embedded in their asthma care. By adding corticosteroid administration to their established protocol, time to steroid administration decreased from 72 to $28 \mathrm{~min}(P<.001)$. We hypothesize that factors that may have contributed to their success included their familiarity with nurse-driven asthma treatment protocols, their high adherence to the protocol, or adequate statistical power that allowed them to find improvements in subject-oriented outcomes.

Our order set was initiated by triage nursing in $22 \%$ of eligible subjects in our post-protocol cohort. We speculate that suboptimal protocol initiation could have been due to lack of staff awareness, misunderstanding of the criteria for protocol initiation, and refusal among the nursing staff to initiate standing orders. The low buy-in may also reflect a behavior that was not surveyed: nursing staff communicating protocol eligibility to the physician staff. Thus, although nurseinitiated, the physician actually entered the orders, thereby underestimating adherence to the protocol. To improve adherence, before launching the protocol, signs were posted in the triage area, e-mails were sent to the nursing staff, and 
announcements regarding the protocol were made in the nursing staff's pre-shift huddle. During protocol initiation, AGM and the emergency department's nursing educators provided one-on-one education with the nursing staff working in triage. Feedback was given to the nursing staff when subjects who were eligible for the protocol were not enrolled. Based on surveys, some triage nurses remained unaware of the protocol during the initial rollout, and some refused to implement the order set, despite understanding the rationale for the protocol. Despite these efforts, other training initiatives could have been considered (eg, requiring online training modules for all emergency department staff, making announcements at emergency department meetings, initiating automatic order sets linked to intake diagnosis codes, and encouraging the RT staff to enter the standing order set during their initial evaluation).

We have gained a greater appreciation for the barriers to implementing this protocol and have developed strategies to overcome them in the next phase of our quality-improvement efforts. For example, lack of nursing education was the primary barrier, as well as nursing reticence in ordering the standing order set. We believe that sharing our experience in implementing our protocol can be helpful to other institutions considering a nurse-driven protocol for pediatric asthma management. To improve accountability, we will work more closely with the nursing administration to develop an incentive structure, but we will also partner with RTs so that the burden does not fall solely on the nursing staff. For example, the RTs working in the emergency department could initiate the order set during their initial evaluation as a backup if the triage nurse did not enter the orders. Providing detailed feedback to the nursing staff could also improve adherence. Although the suboptimal use of the protocol in triage confounds the results of this report, significant improvement in the targeted areas was observed.

Protocol-based care has been shown to be an effective strategy for the initial management of pediatric asthma. Our study reflects the difficulty of incorporating an asthma protocol into a clinical pathway. Our protocol resulted in improvement in all 3 targeted areas despite only $60 \%$ of eligible subjects being enrolled in the protocol and only $22 \%$ of subjects having the protocol initiated in triage as was planned. Specifically, the time to corticosteroid administration was improved by $20 \mathrm{~min}$. Although this did not reach statistical significance $(P=.06)$, we believe that this reduction may lead to significant clinical improvements. Enhanced medication administration efficiency and adherence to evidence-based guidelines in the emergency department are important areas to target for improvement, which can result in reductions in hospital admissions, time to clinical improvement, incidences of respiratory failure, and emergency department length of stay. We expect increased protocol utilization in triage to result in further improvements in efficiency outcomes. To this end, more education is needed to increase staff awareness, acceptance, and protocol utilization.

\section{Limitations}

There are several limitations in our study. Only subjects who received continuous albuterol were chosen for inclusion as a marker for status asthmaticus; however, there were no set criteria for continuous albuterol initiation. As a result, it is possible that physician thresholds for continuous albuterol therapy may have differed between pre- and post-protocol groups, which could have affected the results. The lack of a scoring system or objective measures of disease severity between groups limited our ability to measure the protocol's effect on patient-oriented outcomes, such as hospital admission, pediatric ICU admission, and emergency department length of stay. Hospital and pediatric ICU admission criteria were not standardized and varied based upon the attending physician. The low rate of protocol initiation in triage may have resulted in an overestimation of the magnitude of the effect of the protocol on time to treatment. With improved adherence to the protocol, we would expect to see further increases in efficiency outcomes in this patient population. The retrospective nature limited the available data to those recorded in the electronic medical record. Finally, this study was limited to subjects who received continuous albuterol in a single pediatric emergency department; therefore, the results are not generalizable to all pediatric patients with asthma or all pediatric emergency departments.

\section{Conclusions}

In summary, an asthma protocol resulted in improved adherence to NIH guidelines in children with status asthmaticus and improved efficiency in the administration of rescue bronchodilator and systemic corticosteroid therapy. Future research should examine the role of protocol-based care in the emergency department for patients presenting with status asthmaticus who require treatment with continuous albuterol, including the incorporation of adjunctive therapies such as heliox, intravenous magnesium, noninvasive ventilation, and high-flow nasal cannula. A randomized controlled trial of protocol-based asthma care in the emergency department may be warranted.

\section{ACKNOWLEDGMENTS}

We thank Ira Cheifetz MD FAARC and Michael A Gentile RRT FAARC (Pediatric Critical Care Medicine, Duke University Medical Center, Durham, North Carolina) and P Brian Smith MD MHSc MPH (Department of Pediatrics-Neonatology, Duke University Medical Center) for critical evaluation of the manuscript. 


\section{Pediatric Asthma Protocol in the Emergency Department}

\section{REFERENCES}

1. Akinbami LJ, Moorman JE, Bailey C, Zahran HS, King M, Johnson $\mathrm{CA}$, et al. Trends in asthma prevalence, health care use, and mortality in the United States, 2001-2010. NCHS Data Brief 2012;(94):1-8.

2. Jones BP, Paul A. Management of acute asthma in the pediatric patient: an evidence-based review. Pediatr Emerg Med Pract 2013; 10(5):1-23.

3. Nievas IF, Anand KJ. Severe acute asthma exacerbation in children: a stepwise approach for escalating therapy in a pediatric intensive care unit. J Pediatr Pharmacol Ther 2013;18(2):88-104.

4. National Asthma Education and Prevention Program. Expert Panel Report 3 (EPR-3): Guidelines for the Diagnosis and Management of Asthma-Summary Report 2007. J Allergy Clin Immunol 2007;120(5 Suppl):S94-S138

5. Bhogal SK, McGillivray D, Bourbeau J, Benedetti A, Bartlett S, Ducharme FM. Early administration of systemic corticosteroids reduces hospital admission rates for children with moderate and severe asthma exacerbation. Ann Emerg Med 2012;60(1):84.e3-91.e3.
6. Zemek R, Plint A, Osmond MH, Kovesi T, Correll R, Perri N, Barrowman N. Triage nurse initiation of corticosteroids in pediatric asthma is associated with improved emergency department efficiency. Pediatrics 2012;129(4):671-680.

7. Carroll CL, Sekaran AK, Lerer TJ, Schramm CM. A modified pulmonary index score with predictive value for pediatric asthma exacerbations. Ann Allergy Asthma Immunol 2005;94(3):355-359.

8. Miller A, Mausert S, Dunn M, Eakins K, Thalman JJ, Davies J, et al. Continuous albuterol therapy in a pediatric academic emergency department (abstract). Respir Care 2011;56(10):1698.

9. Rowe BH, Spooner C, Ducharme FM, Bretzlaff JA, Bota GW. Early emergency department treatment of acute asthma with systemic corticosteroids. Cochrane Database Syst Rev 2001;(1): CD002178.

10. Qazi K, Altamimi SA, Tamim H, Serrano K. Impact of an emergency nurse-initiated asthma management protocol on door-to-firstsalbutamol-nebulization-time in a pediatric emergency department. J Emerg Nurs 2010;36(5):428-433. 